\title{
PREVISÃO DO VENTO UTILIZANDO O MODELO ATMOSFÉRICO WRF PARA O ESTADO DE ALAGOAS
}

\author{
DIOGO NUNES DA SILVA RAMOS, ROBERTO FERNANDO DA FONSECA LYRA E \\ ROSIBERTO SALUSTIANO DA SILVA JÚNIOR
}

Universidade Federal de Alagoas, Instituto de Ciências Atmosféricas (UFAL/ICAT), Maceió, AL, Brasil

diogonsramos@gmail.com,roberto.ufal@gmail.com,rosibertojr@gmail.com

Recebido Maio de 2012 - Aceito Setembro de 2012

\begin{abstract}
RESUMO
O prognóstico do vento utilizando o modelo atmosférico WRF para o Estado de Alagoas é investigado neste trabalho. Com medições anemométricas realizadas no período de agosto/2007 a julho/2008, as previsões foram validadas estatisticamente nas estações situadas nas três mesorregiões do Estado: Sertão, Agreste e Litoral. Os resultados mostraram que a qualidade da previsão do vento realizada com o WRF é bastante satisfatória, principalmente no período seco do interior alagoano. Os prognósticos do vento na quadra chuvosa no litoral apresentaram bias de 1,77 m.s. ${ }^{-1}$ e RMSE de 3,61 m.s. ${ }^{-1}$, enquanto no período seco estes índices foram $0,98 \mathrm{~m} \cdot \mathrm{s}^{-1} \mathrm{e} 2,99 \mathrm{~m} \cdot \mathrm{s}^{-1}$, respectivamente. No interior, estes indicadores atingiram bias de $-0,2 \mathrm{~m} . \mathrm{s}^{-1} \mathrm{e}$ RMSE de $2,75 \mathrm{~m} . \mathrm{s}^{-1}$ nos meses secos, e bias de $-0,1 \mathrm{~m} \cdot \mathrm{s}^{-1}$ e RMSE de 2,63 m.s $\mathrm{s}^{-1}$ no período chuvoso. Alguns aspectos do modelo WRF precisam ser mais bem testados e avaliados para aperfeiçoar a previsão na quadra chuvosa, principalmente as parametrizações de microfísica de nuvens e de cumulus. Os índices estatísticos obtidos foram equivalentes, e melhores em alguns casos, a outros estudos deste gênero, indicando que o WRF é uma boa ferramenta para previsão do vento.
\end{abstract}

Palavras-Chave: Previsão do Vento, Modelo WRF, Energia Eólica, Alagoas.

ABSTRACT: WIND PREDICTION USING THE WRF MODEL IN THE ALAGOAS STATE, BRAZIL

This paper aim to analyses wind prediction over the Alagoas State (Brazil) using the WRF model. A 1 -yr (August/2007 to July/2008) anemometer collected data is used to analyze wind pattern and the model predictions were statistically validated using stations located in the three State mesoregions: Backlands (Sertão), Rural (Agreste) and Coast (Litoral). The results showed that quality of WRF model prediction proved to be quite satisfactory especially in the interior of the State during the dry season. The wind predictions in the rainy season for coastal zones showed bias of $1.77 \mathrm{~ms}^{-1}$ and RMSE of $3.61 \mathrm{~m} . \mathrm{s}^{-1}$, while in the dry season these ratios were $0.98 \mathrm{~m} . \mathrm{s}^{-1}$ and $2.99 \mathrm{~m} . \mathrm{s}^{-1}$, respectively. In the interior of the State, these indicators reached bias of $-0.2 \mathrm{~m} \cdot \mathrm{s}^{-1}$ and RMSE of $2.75 \mathrm{~m} . \mathrm{s}^{-1}$ for dry months, and bias of $-0.1 \mathrm{~m} \cdot \mathrm{s}^{-1}$ and RMSE of $2.63 \mathrm{~m} \cdot \mathrm{s}^{-1}$ for rainy season. Some aspects of the WRF model must be further tested and analyzed to improve the prediction during the rainy period, especially the parameterizations of cumulus and clouds microphysics. The obtained statistical indexes were equivalent or better, in some cases, if compared to other similar studies, indicating that the WRF is a good tool for wind forecasting.

Keywords: Wind prediction, WRF Model, Wind Energy, Alagoas. 


\section{INTRODUÇÃO}

Com o crescimento socioeconômico no Brasil nestes últimos anos, temas ligados à infraestrutura elétrica aumentam a preocupação diante da alta dependência dos recursos hídricos para geração de energia. Segundo o Balanço Energético Nacional 2011 (MME, 2011), as usinas hidrelétricas correspondem a $75 \%$ da matriz elétrica total. Então é fundamental reduzir esta dominância das hidrelétricas, que apesar do baixo custo por megawatt (MW) gerado, possui uma grande vulnerabilidade às condições climáticas, além de problemas ambientais (Silva et al., 2005). Nesta situação, a utilização de fontes limpas e renováveis de energia além da hídrica, como a eólica, teve um aumento substancial em todo o mundo na sua geração e pesquisa nos últimos 20 anos (UNEP, 2010).

A partir do Atlas do Potencial Eólico Brasileiro (Amarante et al., 2001) e políticas de incentivo do governo federal, como o Programa de Incentivo a Fontes Alternativas de Energia - PROINFA (Dutra e Szklo, 2007), o Brasil tornou-se um referência mundial no investimento e exploração da energia eólica (REN21, 2011). A maior parte deste potencial está nas áreas costeiras da região do Nordeste Brasileiro (NEB), com $75 \mathrm{GW}$, desconsiderando o potencial offshore. Desde então, a capacidade instalada de geração oriundas dos ventos partiu de 74 MW em 2004 a 2.177 MW em 2010, correspondendo a aproximadamente $0,5 \%$ da matriz elétrica brasileira. Este percentual aumentará até o ano de 2020, pois nos leilões de energia realizados até final de 2011 foram contratados pouco mais de 5.700 MW, totalizando mais de $7.300 \mathrm{MW}$, elevando o Brasil a um novo patamar no cenário global de energia eólica (ANEEL, 2012).

De acordo com o Operador Nacional do Sistema Elétrico - ONS, os parques eólicos implantados pelo PROINFA na região Nordeste geraram eletricidade abaixo do previsto em 2011 (ONS, 2012). Esta defasagem da geração elétrica dos parques eólicos geridos pelo PROINFA possui relação com alguns fatores, dentre os quais, aos estudos produzidos naquela época, que estavam em estágio inicial de pesquisa, principalmente na previsão do vento a curto prazo. Castro et al. (2009) discutiram uma metodologia de análise de eficiência produtiva em sistemas de conversão de energia eólica e destacam também a importância de estudos que avaliem esta condição, pois situações de baixa velocidade do vento, variações climáticas, interrupções de energia elétrica e manutenções (preventivas e corretivas) imprevistas na rede são os principais fatores que elevam o custo operacional das fazendas eólicas ao longo dos anos. Estes problemas não são tão graves em alguns empreendimentos construídos mais recentemente, como usinas eólicas nos Estados do Ceará, Rio Grande do Norte e Rio Grande do Sul, que vem apresentando produção acima do estimado (Costa, 2012).
Os estudos de previsão do vento a curto prazo realizados recentemente no Brasil envolvem diversos tipos de técnicas, como inteligência artificial e métodos estatísticos (Rodrigues, 2007, Gonçalves, 2011), sendo o uso de modelos atmosféricos de mesoescala o mais comum. A investigação da previsão do vento sobre o Nordeste Brasileiro é mais presente na literatura por ser a área com maior potencial, e com maior quantidade de usinas eólicas em operação, como no Ceará e Rio Grande do Norte (Camelo, 2007; Cunha, 2008; Lyra, 2008; De Maria et al., 2008). Existem também levantamentos importantes para as demais regiões do país, com técnicas similares aos estudos aplicados no Nordeste, bem como, na identificação de zonas de considerável potencial eólico (Miguel, 2004; Dalmaz, 2007; Machado, 2008; Cruz Segundo, 2009; Pes, 2010; Mendes, 2011; Oliveira e Costa, 2011).

A aplicação da modelagem atmosférica no prognóstico não apenas do vento mas do tempo em geral é muito importante, pois seu uso traz uma série de benefícios para as mais diversas atividades econômicas da sociedade: turismo; agricultura; aviação civil; transporte marítimo; planejamento urbano, entre outros. Por tanto, uma análise estatística para verificação da eficácia destas previsões também é de suma importância. Este tipo de tratamento possibilita a busca de eventuais problemas nas simulações atmosféricas, bem como, indicar em quais aspectos do modelo a previsão do vento é mais eficiente.

Em Alagoas, região foco deste trabalho, ainda não há na literatura um levantamento deste tipo, com a validação de modelo atmosférico usando medições anemométricas confiáveis para um período longo. Portanto, a realização deste estudo é fundamental, pois informações sobre técnicas de previsão do vento a curto prazo no Brasil, principalmente com modelos atmosféricos, dão sempre suporte à comunidade científica em geral.

Este trabalho objetiva descrever a previsão do vento no Estado de Alagoas com aplicação do modelo atmosférico WRF, caracterizando seus padrões através de dados anemométricos de seis estações distribuídas nas três mesorregiões do Estado para a validação estatística dos prognósticos.

\section{MATERIAIS E MÉTODOS}

\subsection{Medições anemométricas}

Os sítios experimentais estudados neste trabalho foram também utilizados na elaboração do Atlas Eólico do Estado de Alagoas - AEEA (Eletrobras, 2008). As seis estações de medição estiveram distribuídas espacialmente nas três diferentes mesorregiões do Estado de Alagoas: Agreste, nos munícipios de Girau do Ponciano e Palmeira dos Índios; Sertão, em Água Branca; e Litoral, com torres instaladas em Maragogi, Feliz Deserto e Roteiro. Conforme informações contidas na Tabela 
1, os dados referentes ao nível de $30 \mathrm{~m}$ para velocidade do vento serão comparados com o previsto pelo WRF, que foi extrapolado pelo perfil logaritmo do vento. Para a direção do vento, o menor nível das torres será aplicado na comparação com o modelo, ou seja, quatro sítios (Água Branca, Girau do Ponciano, Maragogi e Roteiro) em 50m e dois (Palmeira dos Índios e Feliz Deserto) em 70m.

Cada torre tinha instalado um sistema de aquisição de dados (datalogger) modelo CR800-series da Campbell Scientific inc., funcionando com eletricidade gerada através de um painel fotovoltaico, modelo KS-20 da Kyocera. A velocidade do vento foi medida por um anemômetro polar classe I, modelo A100L2, e o sensor de direção do vento utilizado foi o W200P, ambos confeccionados pela empresa Vector Instruments. Os dados foram medidos a cada segundo, e armazenados em médias a cada 10 minutos. Em seguida, realizou-se uma decomposição vetorial da velocidade e direção do vento para cálculo da média horária (Costa e Lyra, 2012). Estes dados anemométricos foram medidos por sensores calibrados de acordo com as normas internacionais de qualidade e segurança das agências reguladoras: IEA - International Energy Agency (Agência Internacional de Energia); IEC - International Electrotechnical Commission (Comissão Internacional de Eletrotécnica).

\subsection{Modelagem atmosférica em mesoescala: WRF}

O modelo atmosférico de mesoescala WRF, acrônimo de Weather Research and Forecasting Model, é um modelo não hidrostático de previsão numérica de tempo e clima. Seus conceitos físicos são baseados na conservação de massa, explicitando as variações de energia, momentum e umidade do ar. Maiores detalhes quando a estrutura numérica e computacional do modelo podem ser obtidas em Skamarock et al. (2008).

A Figura 1a indica a posição geográfica dos dois domínios configurados no WRF versão 3.2, lançado em abril de 2010. A Figura 1 b apresenta a localização dos sítios estudados no mapa, com indicação também da altitude do Estado de Alagoas. O primeiro domínio possui resolução espacial de $20 \mathrm{~km}$, enquanto o segundo de $5 \mathrm{~km}$. O domínio 1 (D1) abrange parte da região do Nordeste Brasileiro (NEB) e Oceano Atlântico, enquanto o domínio 2 (D2) compreende o Estado de Alagoas. A validação do modelo WRF se deu a partir dos pontos de grade contidos em D2, que foram extraídos para os sítios através de rotinas computacionais geradas em Shell Script, GrADS (http://www. iges.org/grads) e Fortran 90.

Como dados de entrada de WRF, utilizaram-se reanálises do NCEP FNL (Final Analysis) com resolução espacial de

Tabela 1 - Informações geográficas das torres anemométricas de cada local com suas respectivas alturas de medição da velocidade (VV) e direção do vento (DD).

\begin{tabular}{|c|c|c|c|c|}
\hline Município & Coordenadas Geográficas & $\begin{array}{l}\text { Altura da } \\
\text { Torre (m) }\end{array}$ & $\begin{array}{c}\text { Nível } \\
\text { VV(m) }\end{array}$ & $\begin{array}{c}\text { Nível } \\
\text { DD (m) }\end{array}$ \\
\hline Água Branca & $09^{\circ} 14^{\prime} 59,10^{\prime \prime} \mathrm{S} ; 37^{\circ} 56^{\prime} 33,29^{\prime \prime} \mathrm{O} ; 738 \mathrm{~m}$ & 50 & $30-50$ & 50 \\
\hline Feliz Deserto & $10^{\circ} 16^{\prime} 00,91 " \mathrm{~S} ; 36^{\circ} 18^{\prime} 36,29^{\prime \prime} \mathrm{O} ; 36 \mathrm{~m}$ & 100 & $30-70-100$ & $70-100$ \\
\hline Girau do Ponciano & $09^{\circ} 45^{\prime} 58,50 " \mathrm{~S} ; 36^{\circ} 47^{\prime} 06,43^{\prime \prime} \mathrm{O} ; 410 \mathrm{~m}$ & 50 & $30-50$ & 50 \\
\hline Maragogi & $08^{\circ} 59^{\prime} 08,16^{\prime \prime} \mathrm{S} ; 35^{\circ} 12^{\prime} 02,16^{\prime \prime} \mathrm{O} ; 40 \mathrm{~m}$ & 50 & $30-50$ & 50 \\
\hline Palmeira dos Índios & $09^{\circ} 18^{\prime} 19,08^{\prime \prime S} ; 36^{\circ} 41^{\prime} 41,86^{\prime} \mathrm{O} ; 649 \mathrm{~m}$ & 100 & $30-70-100$ & $70-100$ \\
\hline Roteiro & $09^{\circ} 56^{\prime 2} 29,62^{\prime \prime S} ; 35^{\circ} 58^{\prime} 32,66^{\prime \prime} \mathrm{O} ; 40 \mathrm{~m}$ & 50 & $30-50$ & 50 \\
\hline
\end{tabular}

b)

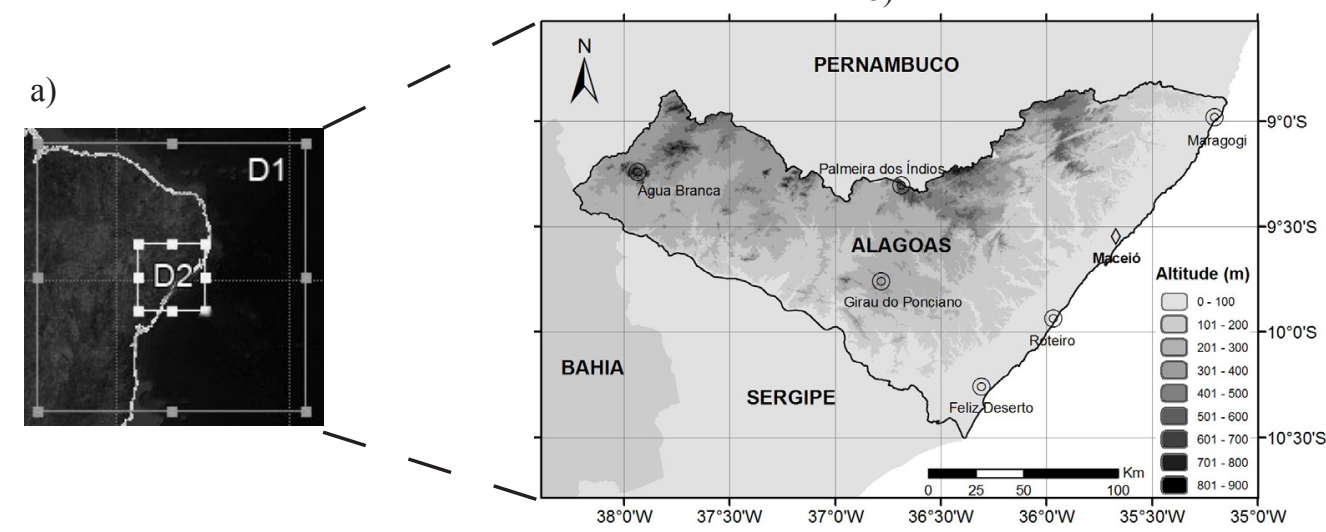

Figura 1 - Representação dos domínios configurados no modelo WRF (a) e localização geográfica dos sítios anemométricos estudados no Estado de Alagoas (b). 
$1,0^{\circ} \times 1,0^{\circ}$ (aproximadamente $111 \mathrm{~km}$ ), e temporal de 6 horas para cada ponto de grade. A composição física da superfície terrestre foi configurada através da topografia USGS (United States Geological Survey - http://www.usgs.gov) e vegetação MODIS (Moderate Resolution Imaging Spectroradiometer http://www.modis.gsfc.nasa.gov), ambos com resolução de 30 segundos, ou $925 \mathrm{~m}$.

As parametrizações físicas utilizadas foram: Microfísica - Purdue Lin (Lin et al., 1983); Superfície do Solo - Noah Land-Surface-Model (Chen e Dudhia, 2001); Camada Limite Atmosférica-ACM2 (Pleim, 2007); Cumulus - Grell-Devenyi (Grell e Devenyi, 2002); Camada Limite Superficial - Teoria da Similaridade de Monin-Obukhov (Monin e Obukhov, 1954); Radiação Onda Longa e Radiação Onda Curta-Rapid Radiative Transfer Model (Mlawer et al., 1997); Turbulência Atmosférica - Mellor-Yamada (Mellor e Yamada, 1974).

As rodadas do modelo foram individuais para cada dia no período estudado, com duração de 30 horas, sendo as primeiras 6 horas de spin-up correspondentes ao dia anterior, que representa o tempo para ajuste das simulações. Através de processamento automatizado por algoritmos em shell script, o WRF executou os 365 dias de agosto/07 a julho/08 em aproximadamente 90 dias.

As previsões do WRF possuíram como saída padrão a velocidade e direção do vento no nível de 10 metros de altura. Portanto, optou-se pelo método da extrapolação vertical do perfil logaritmo do vento (Equação 1) para a menor altura das medições da intensidade do vento, $30 \mathrm{~m}$. Este procedimento foi adotado uma vez que as interpolações obtidas no GrADS superestimaram os prognósticos nas avaliações iniciais deste estudo, sendo o referido perfil logaritmo mais eficiente nestas análises preliminares. No entanto, a direção do vento do modelo foi interpolada verticalmente para os menores níveis das torres de cada local utilizando o GrADS. A aplicação deste método de extrapolação implica em considerar que a atmosfera possui um regime de estabilidade neutra.

$$
u_{z}=\left(\frac{u_{*}}{k}\right) \cdot \ln \left(\frac{Z}{z_{o}}\right)
$$

Onde $\mathrm{u}_{\mathrm{z}}$ - velocidade do vento do WRF no nível desejado $\left(\mathrm{m} . \mathrm{s}^{-1}\right) ; \mathrm{u}^{*}$ - velocidade de fricção simulada no modelo WRF $\left(\mathrm{m} . \mathrm{s}^{-1}\right) ; \mathrm{k}$ - constante de Von Karman $(\mathrm{k}=0,4) ; \mathrm{z}_{0}$ - comprimento de rugosidade $(\mathrm{m})$.

$\mathrm{O}$ valor de $\mathrm{u}^{*}$ usado foi retirado também do modelo WRF, pois segundo Ramos et al. (2011), esta técnica mostrou-se eficaz na validação com os dados anemométricos, seja em período seco ou chuvoso de Alagoas. O valor de $\mathrm{z}_{0}$ foi atribuído para cada localidade investigada de acordo com o proposto em Oke (1988), sendo fixado para todo período anual. Para isso, foram analisadas fotografias tiradas em campo, e a classificação de rugosidade para cada tipo de superfície: Água Branca e Palmeira dos Índios $=0,20 \mathrm{~m}$ (muitas árvores e/ou arbustos);
Girau do Ponciano $=0,03 \mathrm{~m}$ (áreas de pastagem / pequenos cultivos agrícolas); Maragogi, Feliz Deserto e Roteiro $=0,10$ m (vegetação densa).

A discussão das séries com médias diárias da velocidade do vento (u) observada e prevista, nomeados a partir de agora OBS e WRF, respectivamente, se classificada através dos indicadores mencionados por Ramos (2012): Ótimo $=\mathrm{u} \leq 1$ $\mathrm{m} . \mathrm{s}^{-1}$; Bom $=1<\mathrm{u} \leq 2 \mathrm{~m} \cdot \mathrm{s}^{-1}$; Satisfatório $=2<\mathrm{u} \leq 3 \mathrm{~m} \cdot \mathrm{s}^{-1}$; Ruim $=3<\mathrm{u} \leq 4 \mathrm{~m} \cdot \mathrm{s}^{-1}$; Péssimo $=\mathrm{u}>4 \mathrm{~m} \cdot \mathrm{s}^{-1}$.

\subsection{Termos estatísticos}

Foram avaliados nos prognósticos os valores do viés, raiz do erro médio quadrático, desvio padrão e coeficiente de variação, cujas equações são expostas na Tabela 2 . Tais valores estatísticos serão apresentados de forma mensal, obtidos com séries de dados observados e previstos. Vale ressaltar que o cálculo dos índices estatísticos levou em consideração apenas períodos onde os dados fossem pareados, ou seja, quando não houvesse ocorrência de problemas relacionados à ausência e/ ou falhas no banco de dados.

\section{RESULTADOS E DISCUSSÕES}

\subsection{Média diária da velocidade do vento}

A Figura 2 apresenta a média diária da velocidade do vento observada e simulada para os seis sítios estudados. Para Água Branca, as simulações no período entre novembro a dezembro, correspondente a estação seca, foram classificados como ótimos, ou seja, com desvios do WRF perante as medições inferiores a $1 \mathrm{~m} \cdot \mathrm{s}^{-1}$. Os meses que antecedem esta estação apresentam velocidades entre 8 a $12 \mathrm{~m} \cdot \mathrm{s}^{-1}$ em 30 metros de altura, mostrando seu potencial bastante favorável à prospecção de geração elétrica através da fonte eólica. O período chuvoso, maio a julho, deste sítio apresentou velocidades observadas

Tabela 2 - Índices estatísticos avaliados na previsão do vento do modelo atmosférico WRF. N - Número total de dados; $\mathrm{P}$ - Dado previsto; $\mathrm{O}$ - Dado observado; $\mathrm{u}$ - velocidade do vento.

\begin{tabular}{lcc}
\hline \multicolumn{1}{c}{ ÍNDICE } & EQUAÇÃO & UNIDADE \\
\hline Erro médio (bias) & $B I A S=\frac{1}{N} \sum_{i=1}^{N}\left(P_{i}-O_{i}\right)$ & $\mathrm{m} \cdot \mathrm{s}^{-1}$ \\
Raiz do erro médio quadrático & $R M S E=\sqrt{\frac{1}{N} \sum_{i=1}^{N}\left(P_{i}-O_{i}\right)^{2}}$ & $\mathrm{~m} \cdot \mathrm{s}^{-1}$ \\
Desvio padrão & $\sigma=\sqrt{\frac{1}{N-1} \sum_{i=1}^{N}\left(u_{i}-\bar{u}\right)^{2}}$ & $\mathrm{~m} \cdot \mathrm{s}^{-1}$ \\
Coeficiente de variação & $C_{v}=\frac{\sigma}{B I A S}$ & \\
\hline
\end{tabular}



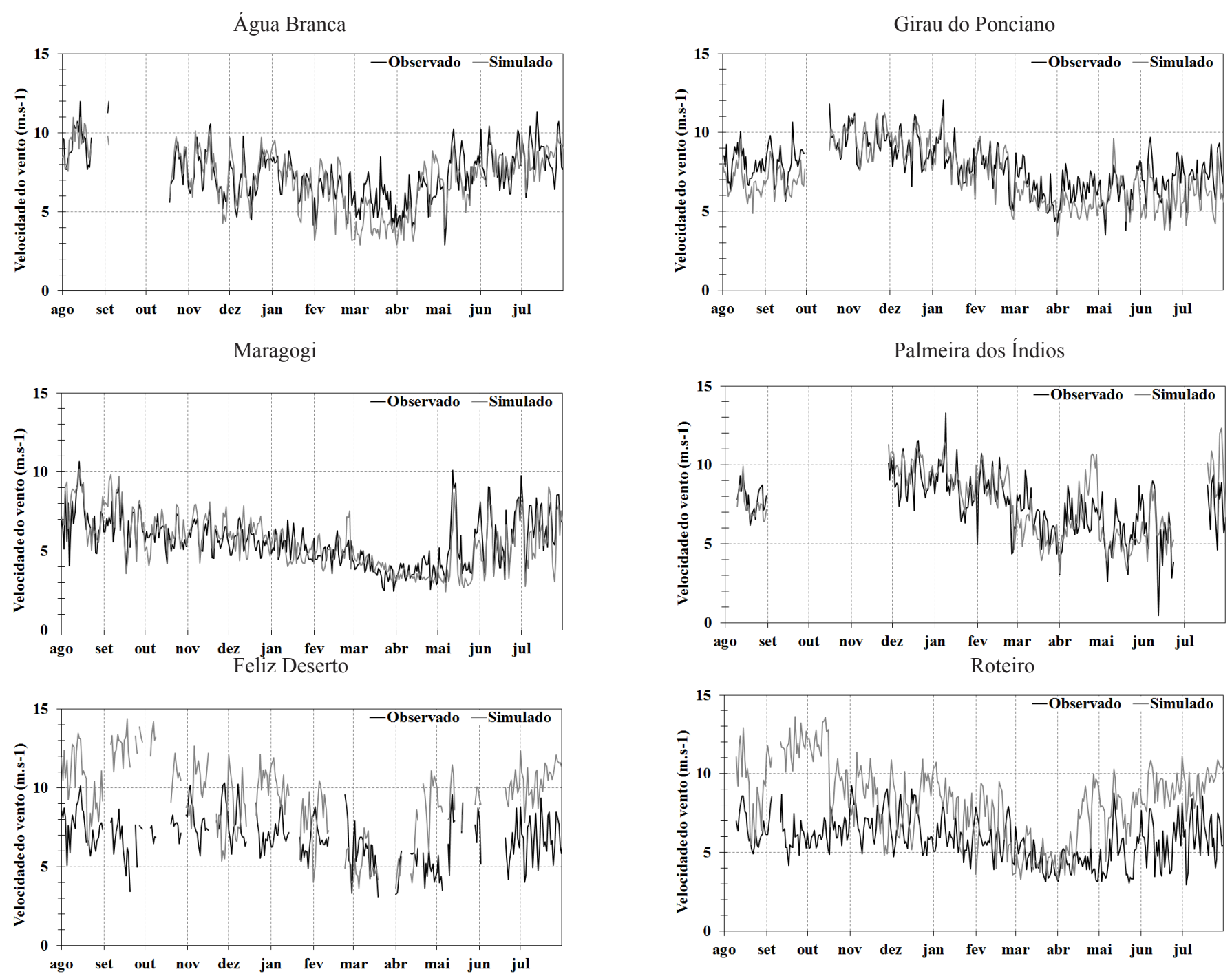

Figura 2 - Média diária da velocidade do vento observada e simulada entre agosto/2007 a julho/2008.

e previstas superiores em até $3 \mathrm{~m} \cdot \mathrm{s}^{-1}$ ao período seco (Costa, 2009; Silva, 2011; Costa e Lyra, 2012). Entre março a abril houve maiores dificuldades do modelo, onde os desvios durante a transição do período seco a chuvoso foram maiores em até $2 \mathrm{~m} \cdot \mathrm{s}^{-1}$, sendo este resultado classificado como de bom a satisfatório.

As previsões em Girau do Ponciano e Palmeira dos Índios são semelhantes na intensidade e comportamento temporal, já que são próximos em aproximadamente $50 \mathrm{~km}$, e as características geográficas locais são semelhantes. Ambos os sítios atingiram os melhores resultados quando comparados aos demais. Desconsiderando os meses de agosto a outubro e março a maio, a diferença média com a velocidade do vento observada no restante do ano foi de $0,2 \mathrm{~m} \cdot \mathrm{s}^{-1}$. O intervalo de magnitude entre $8 \mathrm{a} 12 \mathrm{~m} \cdot \mathrm{s}^{-1}$ acontece em período análogo ao visto em Água Branca. Assim como o sítio sertanejo citado, Girau do Ponciano e Palmeira dos Índios possuem também várias serras, estas com altitudes superiores a $400 \mathrm{~m}$. As condições de terreno de Girau do Ponciano são mais favoráveis que em Palmeira dos Índios em relação ao aproveitamento eólico, pois sua cobertura vegetal é de natureza tipicamente rasteira o ano inteiro, o que implica em menor perda de energia do vento devido à rugosidade superficial. O resultado das previsões do vento para os locais situados no litoral indicam dois períodos da série de médias diárias onde existe uma maior variação da intensidade do vento, entre agosto a outubro e maio a julho. Entre os meses de outubro ao inicio de maio, a velocidade do vento OBS e WRF oscilou entre 2,5 a $8 \mathrm{~m} \cdot \mathrm{s}^{-1} \mathrm{em}$ Maragogi e entre 5 a $10 \mathrm{~m} \cdot \mathrm{s}^{-1} \mathrm{em}$ Feliz Deserto e Roteiro. Houve uma maior dificuldade do WRF em prever com maior precisão os valores máximos da velocidade do vento no período seco e mínimos durante o período chuvoso, condição oposta à visualizada nas médias diárias dos sítios do interior do Estado. Uma hipótese para esta dificuldade nas comparações das previsões com as medições para o litoral é a existência de 
algum efeito extremamente local, que não está sendo possível calcular na resolução espacial do WRF adotada, 5 x $5 \mathrm{~km}$. Por exemplo, os sítios de Feliz Deserto e Roteiro estão situados em zonas com extensas faixas de falésias de até $50 \mathrm{~m}$, o que certamente influencia nos padrões de vento nestes locais, com recirculações do ar sobre este tipo de terreno. A cobertura vegetal também é bastante heterogênea, com presença de canaviais a coqueirais, além de pequenos centros populacionais. Somado a estes aspectos, há ainda a limitação da resolução espacial de topografia e vegetação do modelo WRF, que é de $925 \mathrm{~m}$, implicando em aproximações destes fatores geográficos no litoral, onde a variação de cobertura do solo é mais acentuada. Costa e Lyra (2012) mostram que os padrões do vento nestes sítios litorâneos são caracterizados pela circulação de brisas. É comum também a ação de mecanismos convectivos sobre a região costeira do Nordeste. Segundo o Boletim Climanálise do Centro de Previsão de Tempo e Estudos Climáticos (CPTEC) para o mês de maio de 2008 (CPTEC, 2008), a ocorrência de Distúrbios Ondulatórios de Leste (DOL), na costa leste do NEB, resultou em anomalias positivas da precipitação na região de estudo. Yamazaki e Rao (1977) descrevem o DOL como um evento sinótico que acelera o vento presente na superfície em até $5 \mathrm{~m} . \mathrm{s}^{-1}$, além do sistema ter duração média de quatro dias. Tais configurações do mecanismo meteorológico atuante foram observadas e também captadas nas simulações do WRF com boa acurácia. Papanastasiou et al. (2010) mostraram através do WRF e parametrizações físicas semelhantes às aplicadas neste estudo, como o modelo interpreta os padrões do vento numa região tipicamente caracterizada pela ação de brisas marítimas. Afirmam que durante o dia, as brisas marítimas são responsáveis pela redução da magnitude do vento sobre o continente. Ventos fracos induzem a maiores erros das parametrizações físicas aplicadas no modelo, principalmente nos níveis mais próximos da superfície. Como o presente trabalho realizou a previsão do vento através da sua extrapolação vertical pelo perfil logaritmo do vento usando o $\mathrm{u}^{*}$ simulado no WRF, os períodos de maiores desvios com os dados OBS podem ser explicados pela afirmação dos autores supracitados. Dodla e Ratna (2010) mostram que em momentos de transição climatológica (estação seca para chuvosa), é comum que modelos atmosféricos apresentem uma defasagem na qualidade das simulações. Os meses de março a maio em Água Branca, Girau do Ponciano e Palmeira dos Índios evidenciam este argumento, dividindo a eficiência das simulações entre ótimo (no período seco) e bom (no período chuvoso). Os autores concluem ainda, que os processos termodinâmicos, relacionados às mudanças de cobertura vegetal, podem apresentar resultados de menor qualidade, e assim influenciar na representação numérica da Camada Limite Atmosférica (CLA) do modelo.
O prognóstico do vento, que se trata de uma variável meteorológica de alta frequência temporal, é comumente limitado a aproximações nos termos turbulentos de subgrade, ainda mais quando a previsão é realizada para curto prazo (Pielke, 2002). Então, as séries da velocidade do vento registrada pelos anemômetros em Água Branca, Girau do Ponciano e Maragogi, ao atingirem elevadas intensidades, aumentarão os desvios nas comparações diretas com os prognósticos do modelo WRF. Além disto, Chen et al. (2007) demonstram que confrontar a eficiência de modelos de mesoescala com dados observados em um ponto de grade implica em uma cadeia de limitações de exatidão numérica. Dentre elas, a comparação temporal sofre influência da frequência de registro digital das medições anemométricas, ocorrendo a cada 10 minutos, sendo diferente da escala temporal do WRF, que é horária.

\section{2 Índices estatísticos}

O levantamento estatístico dos padrões de vento é tão importante quanto seu prognóstico. Sendo assim, a Tabela 3 mostra os valores de cada termo estatístico adotado neste trabalho, separados por mês, local e tipo de dado analisado, seja observado ou previsto.

Analisando o modelo WRF sazonalmente para os locais investigados, exceto Feliz Deserto e Roteiro, os meses que apresentaram bias absoluto inferior a $0,5 \mathrm{~m} \cdot \mathrm{s}^{-1}$ estão compreendidos no período seco, entre outubro a fevereiro. Nestes meses citados, o viés médio para Água Branca foi de $-0,18 \mathrm{~m} . \mathrm{s}^{-1}$, Girau do Ponciano com -0,19 m.s ${ }^{-1}$ e Maragogi com $0,21 \mathrm{~m} \cdot \mathrm{s}^{-1}$, enquanto para os demais meses destes locais os valores foram de $-1,05 \mathrm{~m} . \mathrm{s}^{-1},-0,89 \mathrm{~m} \cdot \mathrm{s}^{-1}$ e $0,22 \mathrm{~m} \cdot \mathrm{s}^{-1}$, respectivamente. Os valores médios da velocidade do vento em todo o período estudado, mostrados na Tabela 3, tornam evidente o bom desempenho do WRF, onde os desvios anuais entre previsão versus observação foram: Água Branca $=-0,40$ $\mathrm{m} . \mathrm{s}^{-1}$; Girau do Ponciano $=-0,60 \mathrm{~m} \cdot \mathrm{s}^{-1}$; Palmeira dos índios $=0,22 \mathrm{~m} \cdot \mathrm{s}^{-1}$; Maragogi $=-0,04 \mathrm{~m} \cdot \mathrm{s}^{-1} ;$ Feliz Deserto $=2,32$ $\mathrm{m} . \mathrm{s}^{-1}$ e Roteiro $=2,27 \mathrm{~m} \cdot \mathrm{s}^{-1}$. A dispersão geral das previsões, interpretada por meio do RMSE, indicou valores entre 2,36 a $3,84 \mathrm{~m} . \mathrm{s}^{-1}$, sendo inferior a $3 \mathrm{~m} . \mathrm{s}^{-1}$ apenas aqueles no interior e em Maragogi.

Na região costeira, os índices do bias e RMSE no período seco foram $0,98 \mathrm{~m} \cdot \mathrm{s}^{-1}$ e $2,99 \mathrm{~m} \cdot \mathrm{s}^{-1}$, e na estação chuvosa estes valores foram de $1,82 \mathrm{~m} . \mathrm{s}^{-1} \mathrm{e} 3,61 \mathrm{~m} . \mathrm{s}^{-1}$, respectivamente. Para o interior do Estado os termos estatísticos são melhores, onde nos meses secos o bias e RMSE atingiram valores médios de $-0,17 \mathrm{~m} \cdot \mathrm{s}^{-1} \mathrm{e} 2,75 \mathrm{~m} \cdot \mathrm{s}^{-1}$, enquanto na época chuvosa alcançaram $-0,11 \mathrm{~m} \cdot \mathrm{s}^{-1}$ e $2,63 \mathrm{~m} \cdot \mathrm{s}^{-1}$.

Gonçalves (2011) ao aplicar o modelo ETA para prognóstico do vento em regiões do NEB mostrou valores de 
Tabela 3 - Valores mensais dos índices estatísticos da velocidade do vento observada e simulada.

\begin{tabular}{|c|c|c|c|c|c|c|c|c|c|c|c|c|}
\hline \multirow{3}{*}{ Mês } & \multicolumn{6}{|c|}{ ÁGUA BRANCA } & \multicolumn{6}{|c|}{ GIRAU DO PONCIANO } \\
\hline & \multicolumn{2}{|c|}{$\overline{\boldsymbol{U}}$} & \multicolumn{2}{|c|}{ CV } & \multirow{2}{*}{ BIAS } & \multirow{2}{*}{ RMSE } & \multicolumn{2}{|c|}{$\bar{U}$} & \multicolumn{2}{|c|}{$\mathrm{CV}$} & \multirow{2}{*}{ BIAS } & \multirow{2}{*}{ RMSE } \\
\hline & OBS & WRF & OBS & WRF & & & OBS & WRF & OBS & WRF & & \\
\hline IGC & 8,79 & 8,46 & 0,18 & 0,34 & $-0,33$ & 3,16 & 7,44 & 6,37 & 0,20 & 0,42 & $-1,07$ & 2,67 \\
\hline SET & & , & . & & . & & 7,63 & 6,55 & 0,22 & 40 & $-1,08$ & 2,75 \\
\hline DUT & 7,31 & 7,49 & 0,28 & 0,25 & 0,18 & 2,28 &, 03 & 8,93 & 0,19 & 32 & $-0,10$ & 2,86 \\
\hline so & 93 & 6,73 & 0,34 & 0,33 & $-0,20$ & 2,48 & 23 & 8,99 & 0,2 & 0,34 & $-0,24$ & 3, \\
\hline DEZ & 75 & 6,63 & 0,33 & 0,32 & $-0,12$ & 2,48 & 60 & 8,64 & 0,27 & 0,34 & 0,05 & 3,43 \\
\hline & & 6,46 & 0,32 & 0,31 & & & 12 & 7,69 & 0,29 & 42 & & \\
\hline EV & 86 & 5,41 & 0,35 & 0,42 & -0 & 2,36 & 7,49 & 7,26 & 0,33 & 0,45 & $-0,23$ & 3,37 \\
\hline MA & 5,35 & 3,76 & 0,39 & 0,51 & -1 , & 3 , & 6,12 & 5,43 & 0,39 & 0,58 & $-0,68$ & 3,21 \\
\hline & & 5,2 & 0,36 & 0,46 & & & 01 & 4,85 & 0,31 & & & \\
\hline MAI & 7,05 & 6,26 & 0,27 & 0,31 & -0 & 2,45 & 5,82 & 5,83 & 0,32 & 0,43 & 0,01 & 2,24 \\
\hline JU & 7,55 & 7,29 & 0,21 & 0,36 & -0 & 3,1 & 6,47 & 5,23 & 0,26 & 0,56 & $-1,24$ & 2,89 \\
\hline UL & 3,15 & 7,63 & 0,21 & 0,31 & $-0,50$ & 2,90 & 6,73 & 5,68 & 0,25 & 0,49 & $-1,03$ & 2,72 \\
\hline Médi & 6,88 & 6,48 & 0,30 & 0,36 & $-0,40$ & 2,69 & 7,39 & 6,79 & 0,27 & 0,44 & $-0,60$ & 2,98 \\
\hline
\end{tabular}

\begin{tabular}{|c|c|c|c|c|c|c|c|c|c|c|c|c|}
\hline \multicolumn{7}{|c|}{ MARAGOGI } & \multicolumn{6}{|c|}{ PALMEIRA DOS ÍNDIOS } \\
\hline AGO & 7,10 & 7,52 & 0,28 & 0,30 & 0,42 & 2,53 & 5,90 & 6,02 & 0,23 & 0,25 & 0,12 & 1,67 \\
\hline SET & 6,77 & 7,25 & 0,25 & 0,33 & 0,49 & 2,33 & - & - & - & - & - & - \\
\hline OUT & 5,90 & 6,06 & 0,21 & 0,38 & 0,16 & 2,40 & - & - & - & - & - & 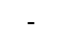 \\
\hline NOV & 6,02 & 6,54 & 0,22 & 0,36 & 0,52 & 2,27 & - & - & - & ـ & - & - \\
\hline DEZ & 5,68 & 5,99 & 0,23 & 0,43 & 0,30 & 2,65 & 7,88 & 7,93 & 0,28 & 0,17 & 0,05 & 2,55 \\
\hline JAN & 5,27 & 5,10 & 0,24 & 0,51 & $-0,17$ & 2,62 & 7,46 & 7,32 & 0,30 & 0,20 & $-0,14$ & 2,36 \\
\hline FEV & 4,93 & 5,16 & 0,28 & 0,54 & 0,23 & 2,60 & 6,86 & 6,90 & 0,36 & 0,23 & 0,04 & 2,53 \\
\hline MAR & 3,80 & 4,12 & 0,34 & 0,57 & 0,33 & 2,29 & 5,55 & 4,59 & 0,38 & 0,43 & $-0,96$ & 2,78 \\
\hline ABR & 3,78 & 3,36 & 0,33 & 0,59 & $-0,42$ & 2,22 & 5,37 & 5,90 & 0,36 & 0,36 & 0,53 & 2,52 \\
\hline MAI & 5,18 & 4,09 & 0,45 & 0,61 & $-1,09$ & 2,55 & 4,22 & 3,95 & 0,40 & 0,32 & $-0,27$ & 1,68 \\
\hline JUN & 5,99 & 5,68 & 0,37 & 0,46 & $-0,32$ & 2,67 & 4,50 & 4,91 & 0,39 & 0,38 & 0,41 & 1,86 \\
\hline JUL & 6,85 & 5,92 & 0,29 & 0,44 & $-0,91$ & 2,62 & 5,49 & 8,11 & 0,26 & 0,32 & 2,67 & 3,77 \\
\hline Média & 5,61 & 5,57 & 0,29 & 0,46 & $-0,04$ & 2,48 & 6,18 & 6,40 & 0,32 & 0,28 & 0,22 & 2,36 \\
\hline & \multicolumn{6}{|c|}{ FELIZ DESERTO } & \multicolumn{6}{|c|}{ ROTEIRO } \\
\hline AGO & 5,79 & 8,54 & 0,24 & 0,35 & 2,76 & 3,79 & 5,89 & 8,16 & 0,23 & 0,42 & 2,27 & 3,94 \\
\hline SET & 5,31 & 10,47 & 0,31 & 0,27 & 5,16 & 5,76 & 5,69 & 10,70 & 0,23 & 0,27 & 5,01 & 5,93 \\
\hline OUT & 6,45 & 9,72 & 0,17 & 0,25 & 3,27 & 4,07 & 5,69 & 9,84 & 0,20 & 0,27 & 4,15 & 5,11 \\
\hline NOV & 6,76 & 7,53 & 0,29 & 0,34 & 0,78 & 3,19 & 6,69 & 7,66 & 0,23 & 0,33 & 0,97 & 3,19 \\
\hline DEZ & 5,96 & 7,97 & 0,29 & 0,29 & 2,01 & 3,47 & 6,01 & 7,55 & 0,28 & 0,32 & 1,54 & 3,63 \\
\hline JAN & 5,62 & 7,65 & 0,29 & 0,27 & 2,03 & 3,00 & 5,49 & 7,35 & 0,26 & 0,31 & 1,87 & 3,04 \\
\hline FEV & 5,44 & 5,84 & 0,35 & 0,46 & 0,40 & 2,83 & 5,51 & 6,15 & 0,30 & 0,42 & 0,64 & 3,01 \\
\hline MAR & 4,85 & 4,30 & 0,34 & 0,53 & $-0,55$ & 2,18 & 4,30 & 4,28 & 0,36 & 0,51 & $-0,02$ & 2,41 \\
\hline ABR & 3,82 & 6,04 & 0,33 & 0,48 & 2,22 & 3,73 & 3,97 & 5,93 & 0,31 & 0,46 & 1,96 & 3,57 \\
\hline MAI & 4,69 & 7,80 & 0,39 & 0,28 & 3,11 & 3,87 & 4,47 & 7,12 & 0,38 & 0,31 & 2,65 & 3,58 \\
\hline JU & 4,82 & 8,00 & 0,35 & 0,3 & 3,18 & 4,25 & 5,15 & 8,30 & 0,32 & 0,36 & 3,15 & 4,44 \\
\hline JUL & 5,20 & 8,68 & 0,29 & 0,31 & 3,50 & 4,26 & 5,59 & 8,68 & 0,29 & 0,31 & 3,11 & 4,26 \\
\hline Média & 5,39 & 7,71 & 0,30 & 0,35 & 2,32 & 3,70 & 5,37 & 7,64 & 0,28 & 0,36 & 2,28 & 3,84 \\
\hline
\end{tabular}

RMSE entre 2,3 a 4,63 m.s. ${ }^{-1}$, e bias entre $-0,43$ a $3,64 \mathrm{~m} \cdot \mathrm{s}^{-1}$. Ainda na região Nordeste, De Maria et al. (2008) avaliando o modelo atmosférico RAMS com diferentes resoluções espaciais, mostraram bias da velocidade do vento entre $-1,6$ a $-3,7 \mathrm{~m} \cdot \mathrm{s}^{-1}$ e RMSE entre 2 a 4,2 m.s. ${ }^{-1}$. Estes últimos autores indicam ainda que a parametrização física de turbulência proposta por Smagorinsky é mais adequada para a região costeira, ao invés do esquema também usado neste estudo, Mellor-Yamada.

\subsection{Direção do vento}

A rosa dos ventos com dados observados e previstos em cada sítio estão contidas na Figura 3. No geral, verificam-se nos dados observados que a direção predominante do vento é Leste, enquanto o WRF indicou uma maior frequência anual em ventos de sudeste. Estes padrões da direção do vento no NEB também foram obtidos por Silva et al. (2004). Somente em Água
Branca a direção predominante do vento observada $\left(135^{\circ}\right)$ ficou mais a Sul do que a simulada $\left(112,5^{\circ}\right)$. Nesta região, a direção predominante é sudeste o ano inteiro devido à canalização do vento sobre o leito do Rio São Francisco comprovada por Ramos (2012). Com exceção de Girau do Ponciano $\left(67,5^{\circ}\right)$ e Roteiro $\left(45^{\circ}\right)$, nas demais localidades a diferença entre as direções predominantes foi de $22,5^{\circ}$. Pode-se afirmar que o WRF simulou melhor a direção do vento em relação à sua magnitude.

\section{CONCLUSÕES}

A previsão do vento, velocidade e direção, realizada pelo modelo atmosférico WRF atingiu os objetivos propostos neste trabalho, com seus índices estatísticos apresentando melhores resultados quando comparado a outros estudos do gênero.

Os valores previstos da velocidade do vento em 30 metros de altura indicaram magnitudes variando de acordo 


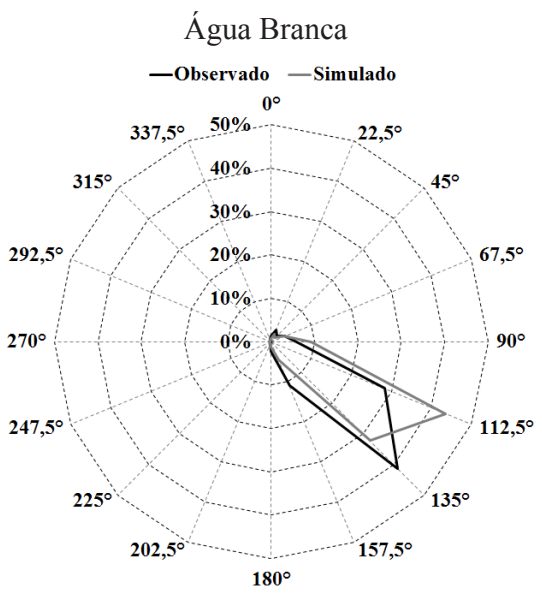

Palmeira dos Índios

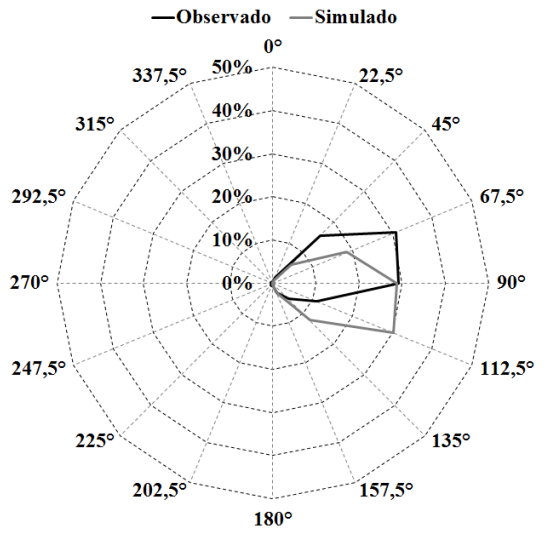

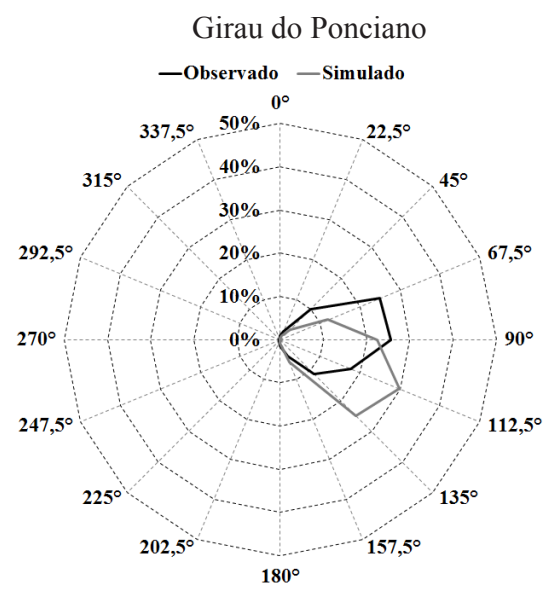

Feliz Deserto

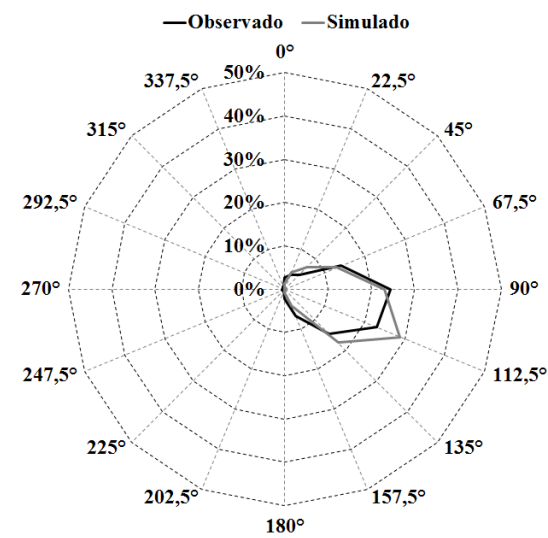

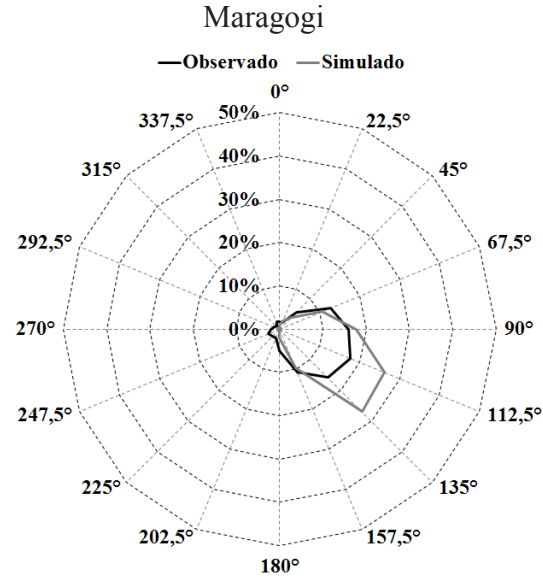

Roteiro

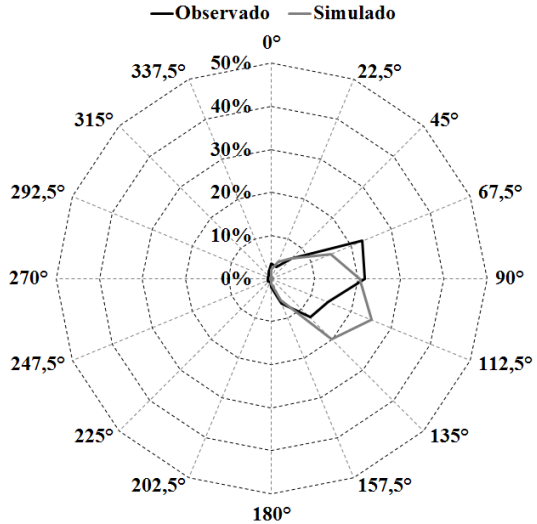

Figura 3 - Direção do vento anual observada e simulada para os locais estudados.

com a mesorregião de Alagoas, com ventos mais intensos no Agreste, seguido do Sertão e Litoral. O prognóstico da direção do vento indicou setores bastante análogos às medições, com ventos de leste a sudeste ao longo do ano. Esta diferença entre os sítios se deve aos fatores locais de topografia e vegetação, como também ação de brisas marítimas e ventos alísios no litoral, brisas vale-montanha no interior e canalização do vento em Água Branca. O modelo WRF conseguiu representar bem estas alterações em escala temporal e espacial, porém houve maior qualidade das simulações quando os locais eram mais distantes do litoral.

Algumas dificuldades foram também encontradas na previsão dos valores extremos, máximos e mínimos da velocidade do vento, e quando o prognóstico realizado aconteceu no período chuvoso, cujos desvios mensais oscilaram entre 3 a 5 m.s $\mathrm{s}^{-1}$ como em Feliz Deserto e Roteiro. Sugere-se que as parametrizações físicas de microfísica de nuvens e cumulus devem ser investigadas para diminuir os erros nas previsões nesta época do ano.

Por fim, as previsões do vento do modelo WRF mostraram-se um instrumento computacional eficaz e importante na realização de levantamentos deste tipo. Sua acurácia na determinação dos padrões médios da velocidade e direção do vento, além do seu desempenho na identificação de fenômenos locais reforça a tese de sua qualidade nos prognósticos de mesoescala.

\section{AGRADECIMENTOS}

Os autores agradecem ao Conselho Nacional de Desenvolvimento Científico e Tecnológico (CNPq) e a Fundação de Amparo a Pesquisa do Estado de Alagoas (FAPEAL) pelo auxílio financeiro concedido.

\section{REFERÊNCIAS BIBLIOGRÁFICAS}

Amarante, O. C. A. et al. Atlas do Potencial Eólico Brasileiro. Brasília: e-dea Techologies, 2001.

ANEEL. Banco de Informação de Geração - BIG, 2012. Disponível em: <www.aneel.gov.br>.Acesso em: 25 fev. 2012. CAMELO, H. N. Estudo numérico do Vento Aracati para caracterização de seu potencial eólico. 2007. 95p. 
Dissertação (Mestrado em Ciências Físicas Aplicadas) Universidade Estadual do Ceará, Fortaleza (CE), 2007.

CASTRO, D. E. et al. Analysis of productive efficiency losses in wind energy conversion systems (WECS). In: 20th International Congress of Mechanical Enginering - COBEM 2009, Anais... Gramado (RS), Brasil, 15 a 20 de Novembro, 2009. CD-ROM.

CHEN, F.; DUDHIA, J. Coupling an advanced landsurface / hydrology model with the Penn State / NCAR MM5 modeling system. Part 1: Model description and implementation. Monthly Weather Review, v. 129, p. 569-585, 2001.

CHEN,J.;HUI,M.C.H.;XU,Y.L.Acomparativestudy of stationary and non-stationary wind models using field measurements. Boundary-Layer Meteorology, v. 122, p. 105-121, 2007. COSTA, G. B. Análise espacial e temporal do vento no Estado de Alagoas. 2009. 116p. Dissertação(Mestrado em Meteorologia) - Universidade Federal de Alagoas, Maceió (AL), 2009.

COSTA, G. B.; LYRA, R. F. F. Análise dos padrões de vento no Estado de Alagoas. Revista Brasileira de Meteorologia, São Paulo, v. 27, n. 31, p. 31-38, 2012.

COSTA, L. Pioneiras, eólicas do PROINFA geram abaixo do previsto no Nordeste. Jornal da Energia - O mundo da eletricidade em tempo real, 2012. Disponível em: <http:// www.jornaldaenergia.com.br>. Acesso em: 25 fev. 2012.

CPTEC. Climanálise - Boletim de Monitoramento e Análise Climática. Centro de Previsão de Tempo e Estudos Climáticos (CPTEC), Instituto Nacional de Pesquisas Espaciais (INPE). Cachoeira Paulista (SP), 47 p. 2008.

CRUZ SEGUNDO, E. I. Estudo de energia eólica para a Ilha de Marajó - PA. 2009. 99p. Dissertação (Mestrado em Meteorologia) - Instituto Nacional de Pesquisas Espaciais (INPE), São José dos Campos (SP), 2009.

CUNHA, E. B. de A. de C. Investigação do potencial eólico de Região Serrana no Ceará. 2008. 86p. Dissertação (Mestrado em Ciências Físicas Aplicadas) - Universidade Estadual do Ceará, Fortaleza (CE), 2008.

DALMAZ, A. Estudo do potencial eólico e previsão de ventos para geração de eletricidade em Santa Catarina. 2007, 196p. Dissertação (Mestrado em Engenharia Mecânica) - Universidade Federal de Santa Catarina (UFSC), Florianópolis (SC), 2007.

DE MARIA, P. H. S.; COSTA, A. A.; SOMBRA, S. S. Modelagem numérica em alta resolução para previsão de geração de energia eólica no Ceará. Revista Brasileira de Meteorologia, v. 23, n. 4, p. 477-489, 2008.

DODLA, V. B. R.; RATNA, S. B. Mesoscale characteristics and prediction of an unusual extreme heavy precipitation event over India using high resolution mesoscale model. Atmospheric Research, v. 95, p. 255-269, 2010.
DUTRA, R. M.; SZKLO, A. S. Incentive policies for promoting wind power producion in Brazil: Scenarios for the Alternative Energy Sources Incentive Program (PROINFA) under the New Brazilian electric power sector regulation. Renewable Energy, v. 33, p. 65-76, 2007.

ELETROBRAS. Atlas Eólico do Estado de Alagoas. Centrais Elétricas Brasileiras S. A. Rio de Janeiro, v. 67 p. 2008.

GONÇALVES, A. R. Refinamento estatístico das previsões de vento do modelo ETA aplicado ao setor eólio-elétrico do Nordeste Brasileiro. 2011. 180p. Dissertação (Mestrado em Meteorologia) - Instituto Nacional de Pesquisas Espaciais (INPE), São José dos Campos (SP), 2011.

GRELL, G. A.; DEVENYI, D. A generalized approach to parameterizing convection combining ensemble and data assimilation techniques. Geophysical Research Letters, v. 29, 2002.

LIN, Y. L.; FARLEY, R. D.; ORVILlE, H. D. Bulk parameterization of the snow field in a cloud model. Journal of Applied Meteorology and Climatology, v. 22, p. 1065-1092, 1983.

LYRA, A. A. Testes de sensibilidade e avaliação das previsões de vento do modelo ETA para Região Nordeste. 2008. 106p. Dissertação (Mestrado em Meteorologia) - Instituto Nacional de Pesquisas Espaciais (INPE), São José dos Campos (SP), 2008.

MACHADO, R. R. Estudo do potencial eólico do Pontal do Abreu - Município de Viamão - RS. 2008. 139p. Dissertação (Mestrado em Engenharia Oceânica) - Universidade Federal do Rio Grande, Rio Grande (RS), 2008.

MELLOR, G. L.; YAMADA, T. A Hierarchy of Turbulence Closure Models for Planetary Boundary Layers. Journal of the Atmospheric Sciences, v. 31, n 7, p. 1791-1806, 1974.

MENDES, C. R. S. Proposta de uma metodologia livre para avaliação de potencial eólico de Minas Gerais. 2011. 112p. Dissertação (Mestrado em Engenharia da Energia) - Universidade Federal de São João Del Rei (UFSJ), São João Del Rei (MG), 2011.

MIGUEL, A. R. F. Análise do potencial eólico para geração de energia elétrica usando dados em microescala de estações climatológicas no Estado de São Paulo. Dissertação (Mestrado em Engenharia Mecânica) - Universidade Estadual de Campinas - UNICAMP, Campinas (SP), 164p. 2004.

MLAWER, E. J. et al. Radiative transfer for inhomogeneous atmosphere: RRTM, a validated correlated-k for the longwave. Journal of Geophysical Research, v. 102, p. 16663-16682, 1997.

MME. Balanço Energético Nacional (BEN) 2011: Ano base 2010 - Resultados Preliminares. Empresa de Pesquisa Energética (EPE), Ministério de Minas e Energia (MME), Governo Federal do Brasil. Rio de Janeiro, p. 52. 2011. 
MONIN, A. S.; OBUKHOV, A. M. Basics laws of turbulent mixing in the surface layer of the atmosphere. Contrib. Geophys. Inst. Acad. Sci., URSS, v. 151, p. 163-187, 1954.

OKE, T. R. Boundary-Layer Climates. $2^{\mathrm{a}}$. ed. Taylor \& Francis Ltda, 460 p. 1988.

OLIVEIRA, J. L.; COSTA, A. A. Estudo de variabilidade do vento em escala sazonal sobre o Nordeste brasileiro utilizando o RAMS: Os casos de 1973-1974 e 1982-1983. Revista Brasileira de Meteorologia, v. 26, n. 1, p. 53-66, 2011.

ONS. Histórico da Operação - Geração de Energia. Operador Nacional do Sistema Elétrico - ONS, 2012. Disponível em: $<$ http://www.ons.org.br>. Acesso em: 25 fev. 2012.

PAPANASTASIOU, D. K.; MELAS, D.; LISSARIDIS, I. Study of wind field under sea breeze conditions; an application of WRF model. Atmospheric Research, p. 102-117, 2010.

PES, M. P. Estudo do impacto das mudanças climáticas no potencial eólico do Estado do Rio Grande do Sul para os períodos de 2010 a 2040 e 2070 a 2100. 2010. 174 p. Dissertação (Mestrado em Meteorologia) - Instituto Nacional de Pesquisas Espaciais (INPE), São José dos Campos (SP), 2010.

PIELKE, R. A. Mesoscale Meteorological Modeling. 2a . ed. San Diego, California: Academic Press, v. 78, 2002.

PLEIM, J. E. A combined local and non-local closure model for the atmospheric boundary layer. Part 1: Model description and testing. Journal of Applied Meteorology and Climatology, p. 1383-1398, 46, 2007.

RAMOS, D. N. S. Mapeamento eólico do Estado de Alagoas utilizando ferramentas computacionais e dados observados. 2012. 130 p. Dissertação (Mestrado em Meteorologia) - Universidade Federal de Alagoas (UFAL), Maceió (AL), 2012.

RAMOS, D. N. S. LYRA, R. F. F.; SILVA JÚNIOR, R. S.; SILVA, A. R. Validação da velocidade do vento do modelo WRF com uso da velocidade de fricção ${ }^{*}$ simulada. Revista Ciência \& Natura, v. Especial, p. 247-250, Santa Maria (RS), 2011.
REN21. Renewables 2011 Global Report Status. GSU. Paris, p. 116. Disponível em <http:// www.ren21.net>. Acesso em: 08 setembro 2011, 2011.

RODRIGUES, G. C. Utilização de redes neurais para previsão de ventos no horizonte de $\mathbf{2 4}$ horas. 2007. 119p. Dissertação (Mestrado em Ciências em Engenharia Elétrica) - Universidade Federal do Rio de Janeiro (UFRJ), Rio de Janeiro (RJ), 2007.

SILVA, A. R. Caracterização do vento e potencial eólico no Estado de Alagoas. 2011. 95p. Dissertação (Mestrado em Meteorologia) - Universidade Federal de Alagoas (UFAL), Maceió (AL), 2011.

SIlvA, B. B.; AlveS, J. J. A.; CAVAlCANTI, E. P.; VENTURA, E. D. Variabilidade espacial e temporal do potencial eólico da direção predominante do vento no Nordeste do Brasil. Revista Brasileira de Meteorologia, v. 19, n. 2, p. 189-202, 2004.

SILVA, N. F.; ROSA, L. P.; ARAÚJO, M. R. The utilization of wind energy in the Brazilian electric sector's expansion. Renewable \& Sustainable Energy Reviews, v. 9, p. 289309. 2005

SKAMAROCK, W. C.; KLEMP, J. B.; DUDHIA, J.; GILL, D. O.; BARKER, D. M.; DUDA, M. G.; HUANG, X. -Y.; WANG, W.; POWERS, J. G. A description of the Advanced Research WRF Version 3. National Center for Atmospheric Research (NCAR). Boulder, Colorado (EUA), p. 125. 2008.

UNEP. Global trends in sustainable energy investiment 2010. United Nations Enviroment Programme (UNEP), Organização das Nações Unidas (ONU). Nova Iorque, p. 61. 2010.

YAMAZAKI, Y.; RAO, V. B. Tropical cloudiness over the South Atlantic Ocean. Journal of Meteorological Society of Japan, v. 55, n. 2, p. 205-207, 1977. 